\title{
Estimation of the coefficient of friction in partial slip contacts between contacting Nickel superalloys
}

\author{
R.M.N. Fleury ${ }^{\mathrm{a}, *}$, R.J.H. Paynter ${ }^{\mathrm{a}}$, D. Nowell ${ }^{\mathrm{a}}$ \\ ${ }^{a}$ Department of Engineering Science, University of Oxford, Parks Road, OX1 3PJ, Oxford, UK
}

\begin{abstract}
Standard methods for measuring the coefficient of friction between two objects are not appropriate for partial slip contacts, since less damage occurs at the interface. A discussion on the different methods for measuring the coefficient of friction in partial slip contacts is carried out. Results of the coefficient of friction between Nickel superalloys, measured by a non-sliding technique, are presented. The effect of crystal orientation of single-crystals on the friction coefficient was found to be small. A study for using the energy dissipated in two-dimensional partial slip contacts is also presented. An approach for measuring the friction coefficient using the dissipated energy is proposed and the challenges and difficulties in measuring friction through the dissipated energy at the contact are discussed.
\end{abstract}

Keywords: friction; fretting; partial slip; contact mechanics

\section{Introduction}

Fretting fatigue occurs primarily in partial slip contacts. Although the surface wear in partial slip is not as significant as in gross sliding contacts, the small slip region in the contact may accelerate crack initiation and propagation, reducing the fatigue life of components. Vingsbo and Söderberg [1] have shown through their map of different fretting regimes that wear may inhibit crack initiation in gross sliding contacts, when material is removed before cracks can initiate. In partial slip contacts, on the other hand, cracks are more likely to initiate and propagate. One of the main difficulties in analysing partial slip problems is that the coefficient of friction is generally unknown inside the contact zone. Crack initiation is governed by the overall surface stress which is, of course, affected by the normal and shear tractions; and the latter are directly dependent on the coefficient of friction at the interface. Incomplete partial slip contacts generally have a central stick zone, where slip does not occur, and where the surface damage is often negligible. It is clear from experiments that the surfaces are significantly less damaged under partial slip when compared to gross slip cases. It has therefore been suggested that the coefficient of friction in a partial slip contact is different from that measured under gross sliding [2], and that in order to accurately

\footnotetext{
${ }^{*}$ Corresponding author. Tel: +4401865273906

Email address: rodolfo.fleury@eng.ox.ac.uk (R.M.N. Fleury)
} 
predict the fretting fatigue life of components it is first necessary to obtain a good estimate of the coefficient of friction in the areas of partial slip.

The traditional method of measuring friction is by measuring the shear load necessary for sliding to occur. This assumes that the friction force depends on the normal force applied, $P$, and a coefficient of friction, $\mu$, as given by Coulomb's law of friction, $Q=\mu P$. However, if a partial slip fretting contact is subsequently slid in this manner by applying a sudden overload, only the average friction coefficient will be obtained, rather than the slip zone value. Several methods have been suggested to estimate the coefficient of friction in partial slip contacts. Hills and Nowell [2] showed that by calculation of the evolution of the stick zone in a fretting experiment, it is possible to infer the coefficient of friction, $\mu_{n}$, in the slip zones after $\mathrm{n}$ cycles from the measurable mean coefficient of friction, $\bar{\mu}$, during sliding. This gives

$$
\begin{array}{r}
\bar{\mu}=\mu_{n}-\frac{2 Q}{\pi P}\{-2 \sin \alpha+ \\
2 \ln \left[\tan \left(\frac{\alpha}{2}+\frac{\pi}{4}\right)\right], \\
\left.+\frac{P \mu_{n} \alpha}{Q}-\tan \alpha\right\},
\end{array}
$$

where $\cos \alpha=Q / \mu_{n} P, P$ is the applied normal force and $Q$ the applied shear force. This method has been used by several authors for Hertzian geometries and isotropic bodies [3, 4]. Dini and Nowell [5] also extended this method to other geometries. However, Eq. (1) applies only when gross sliding takes place in the first few cycles. Hence some surface damage will occur everywhere within the contact. Vadivuchezhian et al. [6] used a similar technique to measure the effect of variable coefficient of friction on the contact traction. They assumed that the coefficient of friction is a function of the total distance slid by each surface point and used a small gross sliding test to predict the evolution of friction for each point in the partial slip contact. They have also shown that if the slip zone size does not change significantly during the test (e.g. $Q / P$ remains constant), then accurate contact tractions are obtained by assuming a constant coefficient of friction throughout the contact. This coefficient is similar to the asymptotic value of the slip zone coefficient of friction. Several authors investigating the coefficient of friction in small amplitude reciprocating contacts have shown that it tends to converge to a value after a transient period of a certain number of cycles [6-8]. However, it is only adequate to use the asymptotic value of the coefficient of friction if the time scale of the asymptote is very small when compared with the time scale of crack initiation (i.e. the number of cycles for initiation is much greater than the number of cycles needed to obtain a converged value of the coefficient of friction in reciprocating gross sliding tests).

An alternative approach for measuring the coefficient of friction in partial slip contacts was suggested by Reina et al. [9]. This method consists of varying the location of the slip zone inside the contact, such that every point in the contact slips at some instant during the loading cycle, but at each instant there is a zone in the contact which is stuck. Hence, the contact is always in partial slip regime, but can be made to displace (or "walk") along the specimen. Less surface damage is observed in this approach than in the one suggested Hills and Nowell [2], since it is not necessary to reach the sliding limit of the contact pair. Instead, the coefficient of friction may be obtained from the shear force which causes transition from stationary behaviour to rigid body motion of the pad. Alternatively, and more practically, an estimate may be obtained from the displacement, 


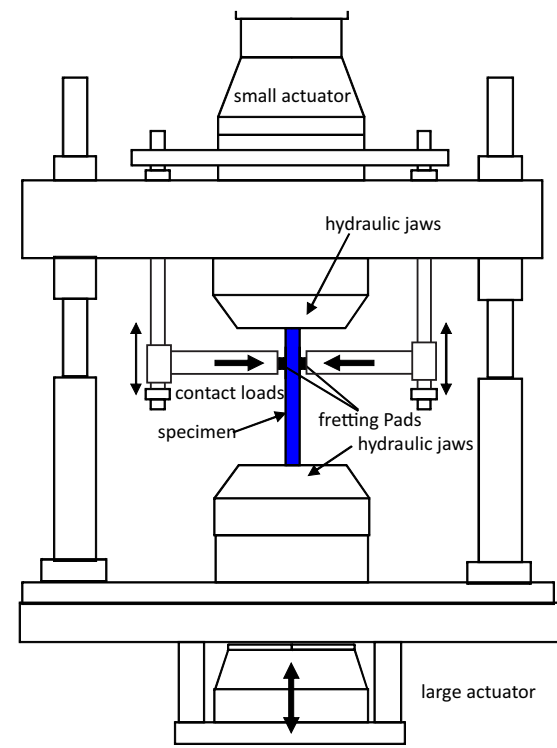

(a) шщщщшщ

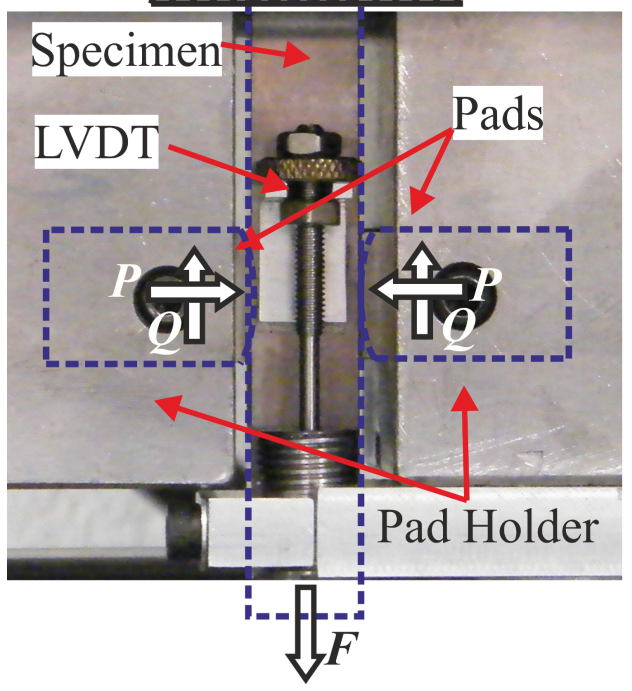

(b)

Figure 1: (a) Schematic representation of the in-line fretting rig; (b) LVDT and pads in the "in-line" rig.

$\Delta s$, of the pad during each load cycle. This approach has been used to measure the coefficient of friction between similar polycrystalline nickel superalloys and for single crystal Nickel superalloy pads pressed against polycrystalline specimens. The results are presented in this paper.

Many authors have also correlated the energy dissipation concept with damage. Fouvry et al. [10] have associated the energy dissipation in gross slip contact problems with the wear rate. It has been shown that axi-symmetric contact problems, e.g. two spheres in contact, have a finite constant displacement at any point sufficiently far from the contact region [11]. Following the work of Mindlin, Fouvry et al. [12] have obtained an analytical solution for the ratio between the energy dissipated and the total energy available. Using the analytical solution for the energy dissipated in a spherical contact problem given by Fouvry et al. [13], Pasanen et al. [14] have presented an energy approach for calculating the energy dissipated that works for the three slip regimes; partial, mixed and gross slip. The approach used in [13, 14] depends largely on the fact that for a contact between two spheres, or a sphere on a plane, the displacement at any point far enough from the contact region is finite and converges to a value which can be obtained analytically. In a two-dimensional half-plane problem this is not the case. The solution of the displacement has a logarithmic form and tends to infinity as the distance from the contact centre increases. However, the work done by each point at the interface due to the slip and shear tractions ought to match the total energy dissipated. Therefore, a study of the energy dissipated at the contact interface of 2D Hertzian contacts will also be presented, together with a discussion of possible energy based methods for plane contact problems. 


\section{The "walking pad" friction test}

\subsection{Experimental procedure}

The "walking pad" tests were conducted using the fretting fatigue experimental rig with two in-line actuators, as illustrated in Fig. 1a. An LVDT sensor was used to measure the relative net displacement between the centre of the contact and the pads. The armature was attached to the specimen at the centre of the contact and the body was attached to the pad holder (Fig. 1b). During the test, the pads are pressed onto the specimen with a constant normal force $P=2.5 \mathrm{kN}$, resulting in a peak contact pressure $p_{0} \approx 80 \mathrm{MPa}$ which is below $1 / 10$ of the yield limit of Nickel superalloys. Hence, no plastic deformation due to the normal contact pressure occurred. Then, a cyclic bulk tension, $\sigma$, is applied to the specimen. The range of the bulk tension cyclic load is raised to a specified amplitude and then kept constant throughout the test. Using the second (small) actuator, the shear force between the pads and the specimen is incrementally increased in a "staircase" fashion. Schematic representations of the loading history and of the applied loads are displayed in Figs. 2a and 2b. In each shear force step, the test ran for about one minute, equivalent to 15 cycles of the bulk tension. The pads remain in partial slip regime at all times and, as the shear load increases, reverse slip starts to occur at the edges of the contact. After a certain value, the stick zone moves from one side to the other in the contact, such that the pads start "walking" along the specimen without ever fully sliding. The higher the coefficient of friction the slower the rate of displacement. During the experiments, the shear loads and displacement data were recorded. With this information, it is possible to calculate an average displacement, $\Delta s$, per load cycle for each shear force increment and, hence, the coefficient of friction.

The solution of the rate of displacement for the "walking" problem can be obtained by quadratic programming [9]. By comparing the quadratic programming solution for different values of coefficient of friction with the experimental data, it is possible to infer the value of the coefficient of friction by interpolation of the experimental results. The coefficient of friction can also be obtained by the shear load limit at which the pads start to walk. However, it is extremely hard to define experimentally the precise moment in which the pads start accumulating a net displacement at the end of each cycle. Examples of the graph that were used to interpolate the coefficient of friction are presented in Figs. 3 and 4. The solid lines represent the displacement per cycle obtained from the quadratic programming solution for each coefficient of friction $\mu$ as the ratio $Q / P$ increases from 0 to 1 . The blue cross points are the displacement per cycle obtained experimentally, with an appropriate normalization. This normalization takes into account the peak pressure, $p_{0}$, the contact semi-width, $a$, and the elastic compliance between the materials, $A^{1}$. Note that for the nickel single-crystal superalloy pads, these variables were obtained by solving the anisotropic contact solution presented by [15]. In the case of the elastic compliance, it can be shown that the constant, $A$, used in isotropic solutions is equivalent to $\operatorname{Re}(\gamma)$ in the equation for the contact pressure of anisotropic bodies given in [15]. Finally, the green circular points (right hand axis) represent the values of the coefficient of friction - obtained by matching the experimental data and the quadratic programming solution for the rate of displacement.

\footnotetext{
${ }^{1}$ For isotropic contacts, the contact elastic compliance is given by $A=\left(\kappa_{1}+1\right) / 4 G_{1}+\left(\kappa_{2}+1\right) / 4 G_{2}$, where $\kappa_{i}$ is the Kosolov's constant, defined as $\kappa=3-4 v$ for plane strain, and $G_{i}$ is the shear modulus of body $i=1,2$.
} 

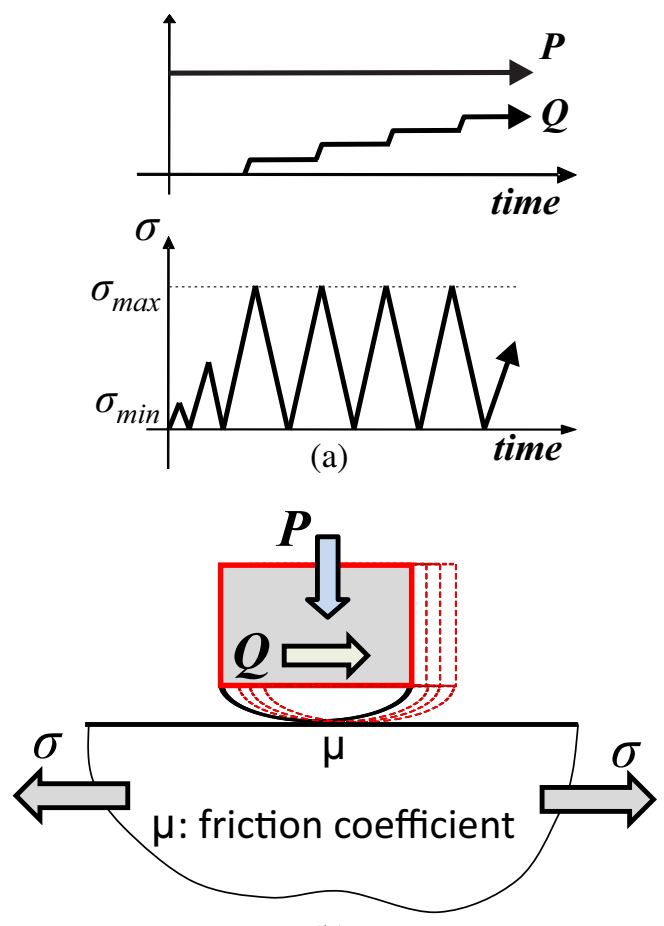

(b)

Figure 2: (a) Load history used in the walking pad tests; (b) Schematic representation of the loading in the "walking pad" test [9].

As second measurement of the coefficient of friction was carried out using the standard gross sliding test. At the end of each "walking" test, the cyclic bulk loading was interrupted and the shear load reduced to zero. Then, the pad carriage actuator was changed to displacement control. A displacement ramp was then applied (i.e. constant actuator velocity), inducing the pad to slide along the interface of the specimen. As the pad was sliding, the shear force, $Q$, was measured, from which the sliding coefficient of friction can be calculated, by averaging the load ration $Q / P$ throughout the sliding phase. Problems associated with this method include the fact that the pad surfaces are significantly damaged during the test. One of the problems of using this method to partial slip contacts is the fact that the pad surfaces are significantly worn out during the test, which does not happen in partial slip. Furthermore, at the beginning of the gross sliding tests, the surfaces of the pad were already slightly damaged, since the tests were carried after the "walking pad" test. Hence, the coefficient of friction measured at the early stages of the gross sliding tests are also not representative of the undamaged partial slip contact where the central slip zone does not experience any surface damage. Note that, although the partial slip test always had a central stick zone, in the "walking pad" test, the stick zone alternates sides during the load cycle and all points on the surface slip, though not all at the same time. This means that the entire contact zone will have some surface damage caused by the micro slip, even before the gross sliding tests begin.

\subsection{Results of the coefficient of friction tests}

The objective of these tests conducted with Nickel superalloys was to investigate the impact of the crystal orientation pads on the coefficient of friction between a polycrystalline part pressed 


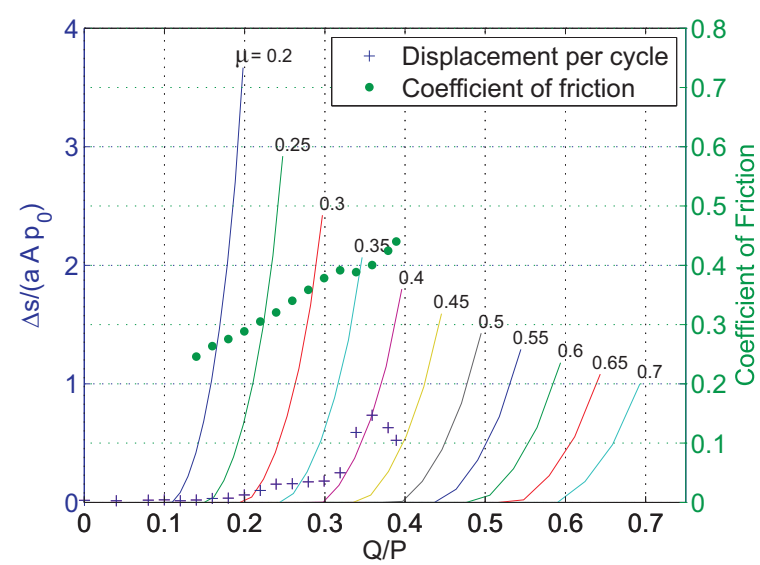

(a)

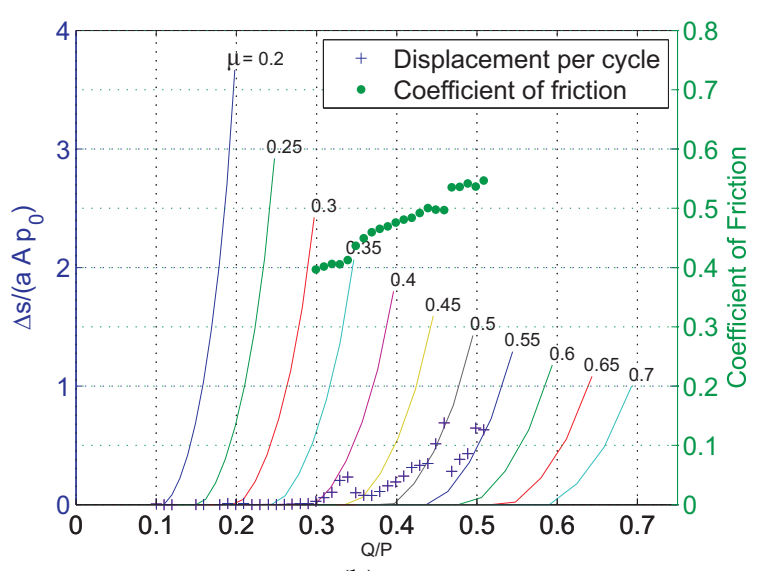

(b)

Figure 3: Coefficient of friction in partial slip contacts between single-crystal and polycrystalline Nickel superalloys. Single-crystal orientation of (a) $\phi_{1}=6.4^{\circ}$; (b) $\phi_{1}=42.7^{\circ}$.

against a single-crystal alloy. This is a relevant problem in the blade to disc assembly at the high pressure turbine in aero-engines. Single-crystal alloys have orthotropic elastic properties and therefore, the contact tractions are dependent on the orientation of the crystal with respect to the geometry of the pads. Suggestions that the coefficient of friction also varies with the crystal orientation has been pointed out as a possible reason for the large scatter in fretting fatigue life observed in tests with single-crystal alloys $[15,16]$. In this work, as well as in [15], we are interested in investigating the impact of varying the crystal orientation whilst keeping one of the crystal principal directions allign with the contact vertical direction. This is misallignement of the the principal crystallographic direction, e.g. $\langle 100\rangle$, with the geometrical $x$-axis is characterised by the angle between these two directions, $\phi_{1}$.

Two typical tests with single-crystal alloy pads are displayed in Fig. 3, one with pads of orientation $\phi_{1}=6.45^{\circ}$, Fig. 3a, and one with pads at $\phi_{1}=42.7^{\circ}$. Note that the angle $\phi_{1}$ represents the deviation of the crystal principal directions with the two Cartesian axes on the plane of the surface of contact [17]. In partial slip regime, the coefficient of friction for single-crystal alloy pads varied significantly as the test was being conducted (Fig. 3a), often with clear steps of constant coefficient of friction (see Fig. 3b). This variation may be associated with the response of the material as the shear load increases. The same behaviour was not seen in the tests with polycrystalline isotropic pads (Fig. 4). The coefficient of friction for tests with similar isotropic pads were also lower than the ones with single-crystal pads. Both tests carried out with similar isotropic pads have shown a coefficient of about $0.20-0.25$.

Two methods were adopted to compare the coefficients of friction for the single-crystal pads in partial slip. The first, takes into account the coefficient of friction in the last cycles before full slip occurs. The second, compares the coefficient of friction for an arbitrary value of $Q / P=0.3$. The coefficient of friction obtained experimentally as a function of the crystallographic orientation of the single-crystal pads are shown in Fig. 5. The results are also listed in Table 1.

In Table 1, tests with the same number and different suffixes ( $a, b$ and $c)$ indicate the same 


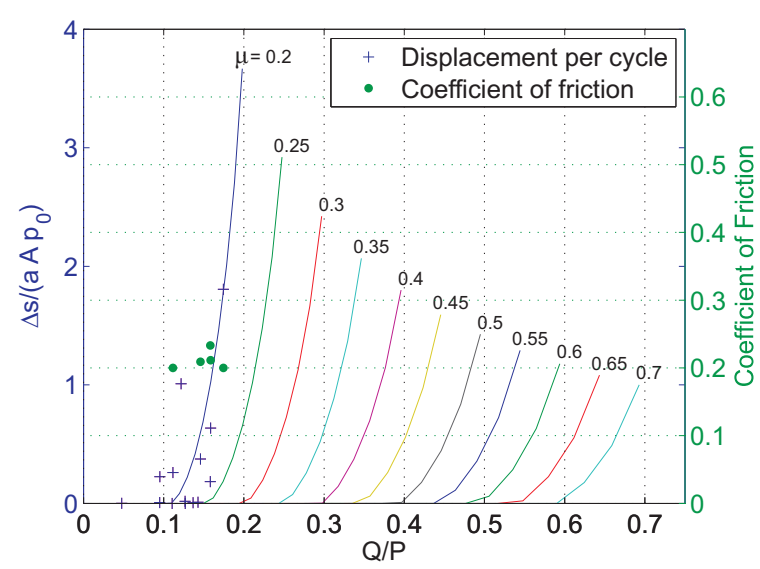

(a)

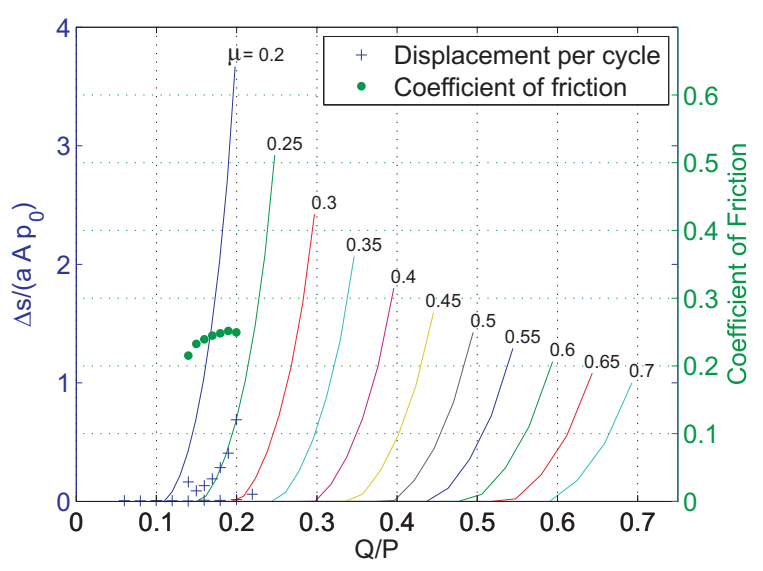

(b)

Figure 4: Coefficient of friction in partial slip contacts between similar polycrystalline Nickel superalloy.

Table 1: Coefficient of friction between single-crystal and polycrystalline Nickel superalloys.

\begin{tabular}{c|c|ccc}
$\begin{array}{c}\text { Test } \\
\text { Number }\end{array}$ & $\begin{array}{c}\text { Crystal } \\
\text { Angle (deg) }\end{array}$ & $\begin{array}{c}\text { Gross } \\
\text { Sliding }\end{array}$ & $\begin{array}{c}\text { Partial Slip } \\
\text { Last Cycle }\end{array}$ & $\begin{array}{c}\text { Partial Slip } \\
\text { Q/P }=0.3\end{array}$ \\
\hline Test00d1a & 6.36 & 0.349 & 0.3477 & 0.3549 \\
Test00d1b & 6.36 & 0.497 & 0.472 & 0.3891 \\
Test30d2a & 9.09 & 0.376 & 0.4216 & 0.3782 \\
Test00d2b & 9.09 & 0.679 & 0.5661 & 0.3786 \\
Test30d1a & 30.0 & 0.586 & 0.5441 & 0.3786 \\
Test30d1b & 30.0 & 0.663 & 0.6186 & 0.3913 \\
Test30d2a & 27.7 & 0.572 & 0.5692 & 0.397 \\
Test45d1a & 42.73 & 0.558 & 0.5417 & 0.3969 \\
Test45d1b & 42.73 & 0.580 & 0.5458 & 0.3888 \\
Test45d2a & 39.09 & 0.465 & 0.4207 & 0.3844 \\
Test45d2b & 39.09 & 0.624 & 0.578 & 0.391
\end{tabular}

pair of pads. Due to the limited number of pads, the surface of the pads were reground and the tests repeated to give an indication of variability between different tests under nominally the same conditions. A large variation between repeated tests was observed for the gross slip and last partial slip cycle value of $\mu$. It is believed that this scatter is due to variations in the roughness of the specimen and pads as they were reground. The three methods listed above resulted in similar coefficients for each test. However, a higher coefficient was measured in gross slip, which could be explained by the increasing wear at the interface of the contact as the test was carried out. Due to the large scatter in the results, it is not possible to correlate near-sliding coefficient of friction with crystal orientation. At a fixed value of $Q / P=0.3$ the results are much more consistent, with all tests presenting a friction coefficient of about 0.38 . Nevertheless, no significant difference in the coefficient of friction was observed as the crystal orientation of the pads changed. 


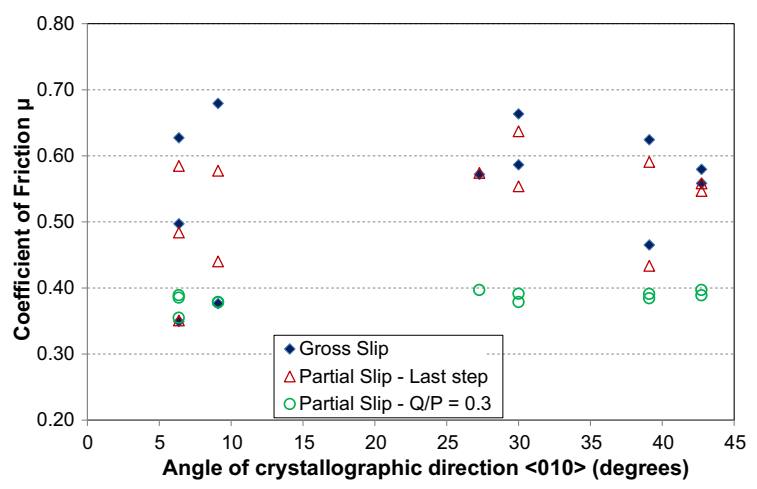

Figure 5: Coefficient of friction between single-crystal and polycrystalline Nickel superalloys as a function of the crystal orientation.

\section{Energy dissipation in partial slip problems}

Following the work of various researches on the frictional energy dissipation in partial slip contacts $[10,13,14]$, carried out primarily on axi-symmetric Hertzian contact problems, we now investigate an energy-based approach for plane contacts.

In a complete load cycle under fretting the displacement is not recovered. That is, if no initial displacement exists between the bodies and an incremental shear load is applied at one of the bodies up to a maximum value $Q_{\max }$ and then decreased back to zero, a residual relative displacement $\delta_{0}$ between the two bodies exists. This phenomenon is caused by the non-linearity introduced to the problem by the presence of friction. According to Coulomb's law of friction [18], the frictional force between two bodies is dependent on the direction of the velocity of movement, being always opposite in sign. In a complete reverse cycle, a hysteresis loop is formed when plotting the relative displacement between the bodies, $\delta$, versus the applied shear force, $Q$, and the area inside the loop is the energy dissipated per cycle. Note that the relative displacement tends to a finite value far from the contact in axi-symmetric problems, but tend to infinity as the observation point moves away from the contact interface in 2D plane contacts. Nevertheless, we propose here that although the displacement is not bounded, far from the contact, the energy dissipated (i.e. are inside the loop) tends to a finite value. The equations for the total energy displacement in 2D Hertzian contacts are derived here and experimental techniques are proposed for measuring the coefficient of friction.

\subsection{Contact tractions and displacements}

Consider the shear traction between two isotropic cylinders. Following the theory of Hertz for spherical contacts, Cattaneo [19] and Mindlin [20] have shown that the presence of a stick zone can be represented by superimposed sliding solutions. The solution was later extended to plane contacts and is presented in [21]. Coulomb friction is assumed at the interface with a coefficient of friction $\mu$. Using the superposition of the solution of the sliding problem and the contribution of the stick zone on the contact traction, the shear traction distribution over a contact region along 


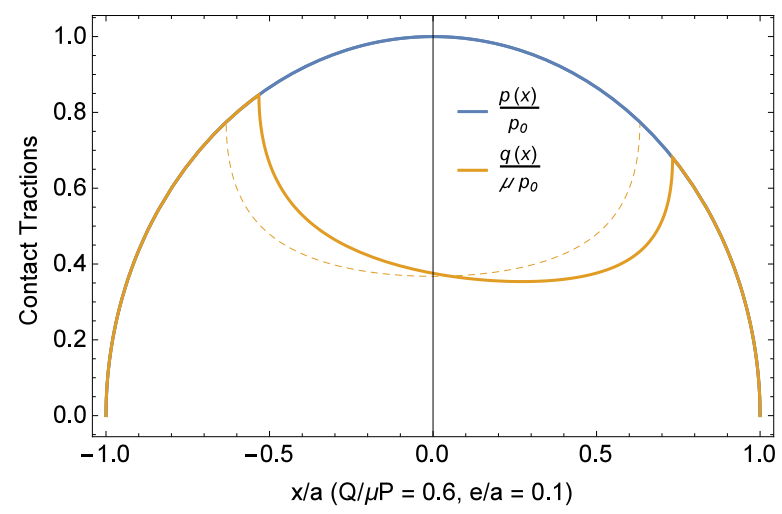

Figure 6: Contact tractions for a contact between two elastically similar isotropic cylinders. Effect of bulk tension on the contact shear traction.

the $x$ axis comprised by the interval $|x| \leq a$, can be written as

$$
q(x)=\mu p_{0} \sqrt{1-\left(\frac{x}{a}\right)^{2}}-q^{\prime}(x), \quad-1 \leq \frac{x}{a} \leq 1
$$

where the peak stress, $p_{0}$, depends on the applied load, $P$, and the contact semi-width, $a,\left(p_{0}=\right.$ $2 P / \pi a)$ and $q^{\prime}(x)$ is the perturbation due to the stick zone. The contact area is a function of the applied normal load, the curvature of the cylinder surfaces and the elastic compliance of the contact pair [21]. The contribution of the central stick zone has the same form as the normal pressure solution and, in the presence of a bulk tension on one of the bodies, where the central stick zone is displaced by an offset, $e$, this is written as [22],

$$
q^{\prime}(x)=\mu p_{0} \frac{c}{a} \sqrt{1-\left(\frac{x-e}{c}\right)^{2}}, \quad-1 \leq \frac{x-e}{c} \leq 1,
$$

where $c / a=\sqrt{1-Q / \mu P}$ and $e / a=\sigma_{0} / 4 \mu p_{0}$. The normal and shear traction distributions for an example contact between two isotropic elastically similar cylinders are presented in Fig. 6.

The singular integral equations for the normal, $h(x)$, and tangential, $g(x)$, relative displacements between two isotropic elastically similar contacting cylindrical bodies, and with one body submitted to a bulk stress tension, are given by [22]

$$
\begin{aligned}
\frac{\partial h(x)}{\partial x} & =\frac{A}{\pi} \int_{-a}^{a} \frac{p(\xi)}{x-\xi} d \xi \\
\frac{\partial g(x)}{\partial x} & =\frac{A}{\pi} \int_{-a}^{a} \frac{q(\xi)}{x-\xi} d \xi-\frac{\sigma_{0}}{E}\left(1-v^{2}\right) .
\end{aligned}
$$

In order to obtain the slip function at the interface, Eq. (4) needs to be solved. Furthermore, if the bodies are elastically similar, the elastic compliance parameter is $A=4\left(1-v^{2}\right) / E$ and the last term of the left hand side of Eq. (4) can be rewritten in terms of the offset of the stick zone, $e / a$. 
The only region of interest for the calculation of energy dissipation is the slip region. This is the only region where energy is dissipated, since slip is zero in the stick zone and the shear traction is zero outside the contact zone. The relative displacement inside the slip zone is given by

$$
\frac{\partial g(x)}{\partial x}=A \mu p_{0} \frac{x-e}{a} \sqrt{1-\left(\frac{c}{x-e}\right)^{2}}, c \leq|x-e| \leq a .
$$

In order to calculate the displacements, Eq. (5) needs to be integrated over the slip zone. The derivation of the relative displacements in the slip zone has been given by several authors and can be found in detail in [17]. This is found to be

$$
\begin{aligned}
\frac{g(x)}{a A \mu p_{0}}= & \frac{1}{2}\left[\frac{x-e}{a} \sqrt{\left(\frac{x-e}{a}\right)^{2}-\left(\frac{c}{a}\right)^{2}}\right. \\
& \left.-\left(\frac{c}{a}\right)^{2} \log \left(\frac{x-e}{c}+\sqrt{\left(\frac{x-e}{c}\right)^{2}-1}\right)\right] .
\end{aligned}
$$

The solution in Eq. (6) is valid for the both regions of slip and also presents the correct answer for the stick zone, which is $g(x)=0$ for $|x-e| \leq c$.

\subsection{Frictional energy dissipation}

The frictional energy dissipated needs to equal the sum of work done by the frictional force at each point in contact. If the shear traction and slip are known throughout the contact interface, the work done at each point of the surface during a complete loading and unloading can be obtained. As it is shown by the Cattaneo-Mindlin solution, as the load is decreased from its maximum value, the stick zone changes in size, but the shear traction at each point in the sliding region remains constant up to the moment when the point stops sliding. Once stuck, no work is done at that point and it will remain at rest until a reverse shear load is sufficient to cause sliding in the opposite direction. The complete load-displacement cycle of an arbitrary point in the sliding zone is illustrated in Fig. 7. The area inside the square in the $q-g$ plot represents the work done by the frictional force at an arbitrary point. The distribution of work done along the contact interface per unit area, $w(x)$, is given by

$$
\begin{aligned}
w(x)= & 4 q(x) g(x) \\
= & 2 a A \mu^{2} p_{0}^{2} \sqrt{1-\left(\frac{x}{a}\right)^{2}}\left[\left(\frac{x-e}{a}\right) \sqrt{\left(\frac{x-e}{a}\right)^{2}-\left(\frac{c}{a}\right)^{2}}\right. \\
& -\left(\frac{c}{a}\right)^{2} \log \left(\frac{x-e}{c}+\sqrt{\left.\left(\frac{x-e}{c}\right)^{2}-1\right)}\right] .
\end{aligned}
$$

The distribution of work done per unit of area is given in Fig. 8 for different offsets of the stick zone $e / a$. Note that the maximum energy dissipated in the contact zone is not at the contact edge, where the peak stress occurs, but at about a distance $0.1 a$ away. This location is likely to be where the most severe wear might occur. The total energy dissipated in the system is given by the integral 


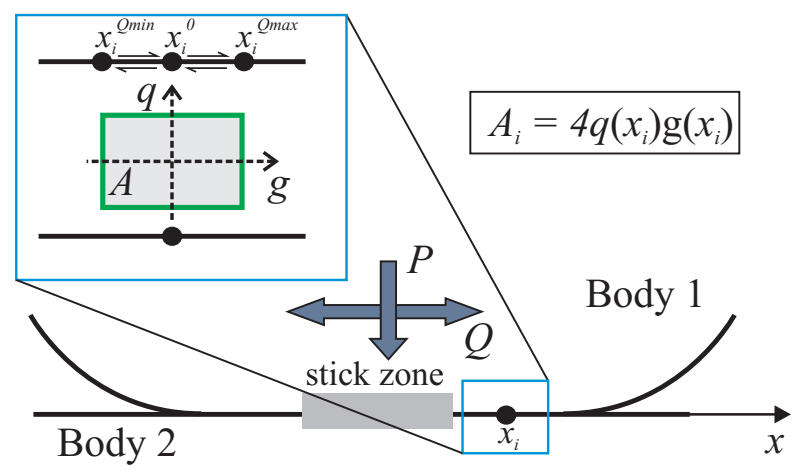

Figure 7: Energy dissipated at each point of the slip zone.

of Eq. (7) over the contact region. In a plane problem of length $z_{0}$ in the $z$ direction (out-of-plane direction), the total work done is given by the following equation,

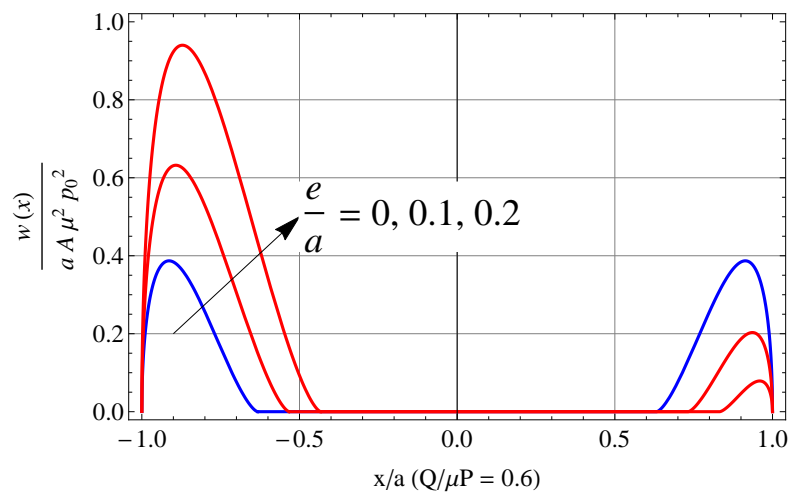

Figure 8: Energy dissipated per unit of area for different values of the stick zone offset $(Q / \mu P=0.6)$

$$
\begin{aligned}
\mathcal{W}=\int_{A} w(x) d A & =\int_{0}^{z_{0}} \int_{-a}^{a} w(x) d x d z \\
& =z_{0} \int_{-a}^{a} w(x) d x
\end{aligned}
$$

\section{Analysis of the energy dissipated}

The frictional energy dissipated in the contact interface during a load cycle can be measured by the hysteresis loop of the applied tangential force, $Q$, and the displacement of an arbitrary point in one of the bodies. Contrary to the work done in spherical contacts, the displacement far from the contact goes to infinity as the observation point moves away from the contact interface. However, if any other dissipative mechanism is negligible, the area inside the hysteresis loop ought to match the frictional energy dissipated in the contact. A finite element (FE) model was used to verify this behaviour and it is displayed in Fig. 9. Equally spaced points in the vertical direction at the centre of the contact were used to verify the frictional energy dissipated through the hysteresis loop as the 


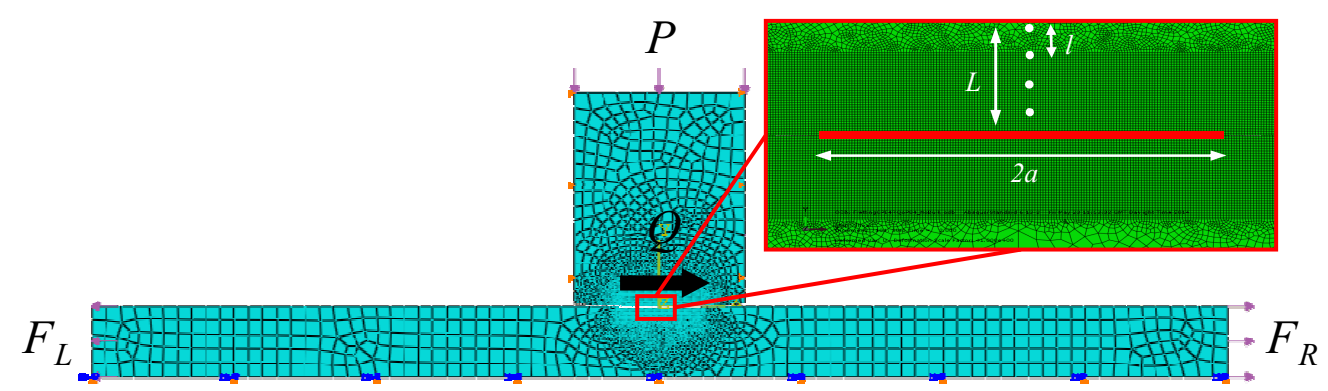

Figure 9: Finite element model of a 2D Hertzian contact fretting problem.

observation point moves away from the contact interface. The load displacement hysteresis loop at those points are displayed in Fig. 10a. The area inside the loop, or frictional energy dissipated, as a function of the distance to the contact interface is shown in Fig. 10b. Note that, despite the increasing value of the displacement of points far from the contact, the area inside the hysteresis loop tends to a constant value after a small zone of perturbation which has approximately the same magnitude as the contact semi-width, $a$. This asymptotic value of the measured energy dissipated is the value given by the frictional energy dissipated at the interface given in Eqs. (7) and (8).

The presence of a bulk load on one of the bodies in contact introduces an offset, $e$, to the stick zone. Because of the non-linearity of the energy dissipated in Eq. (7), the shift of the stick zone changes the total energy dissipated. The increase in work done in one slip zone is greater than the decrease in work done on the other slip zone. One potential benefit of changing the position of the slip zone as a mean of varying the total energy dissipated is that it would be possible to vary the total energy dissipated while keeping the applied load ratio $Q / P$ constant. Note that results obtained with the "walking pad" test suggest that the coefficient of friction between single-crystal and polycrystalline Nickel superalloys vary with the applied load ratio, $Q / P$. Hence, the approach of moving the location of the stick zone reduces the number of variables in the problem, since the cyclic variable, i.e. the bulk force inside one of the bodies, is of little relevance at the interface other than the offset of the stick zone that it causes. By using Eq. (7), the energy dissipated per unit of area along the interface can be obtained and it is shown in Fig. 8. The total energy dissipated, or work done by frictional force, can be obtained by using a numerical integration technique to integrate Eq. (8).

It is also important to correlate the offset, $e$, with the correspondent bulk stress applied in an example problem. Consider a 2D Hertzian contact between a cylinder and a flat plane of unit thickness, $z_{0}=1$. The total work done at the interface as a function of the bulk stress is given in Fig. 11a, where the applied bulk stress $\sigma_{0}$ is normalized by the normal contact peak stress $p_{0}$. The solution was compared with numerical results obtained via finite element analysis and good agreement between the solutions is observed. In a similar manner to the variation of the stick zone offset, variation of the load ratio $Q / P$ varies the work done at the interface (Fig. 11b). However, practical implementation of this approach still depends on the assumption that the coefficient does not change with the $Q / P$ load ratio. The work done per unit of area can again be obtained by making use of Eq. (7), which here can be simplified due to the absence of the stick zone offset. However, large changes in friction do not cause significant difference to the energy expended when 


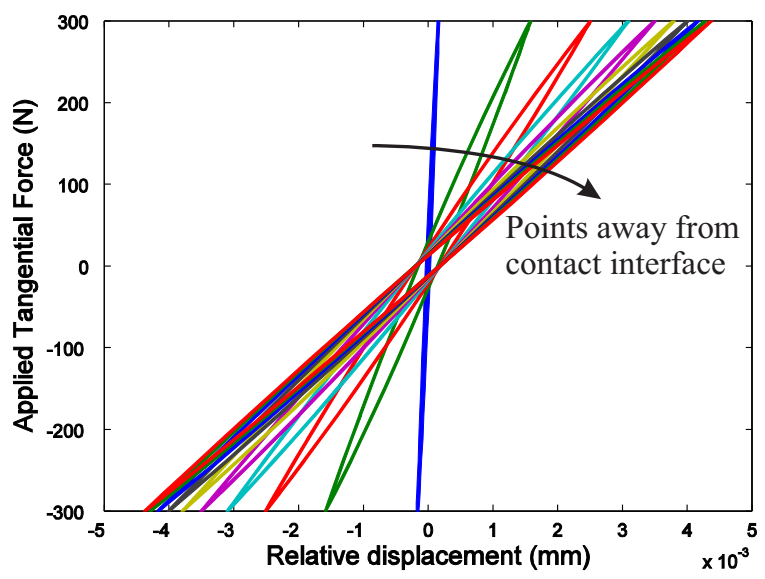

(a)

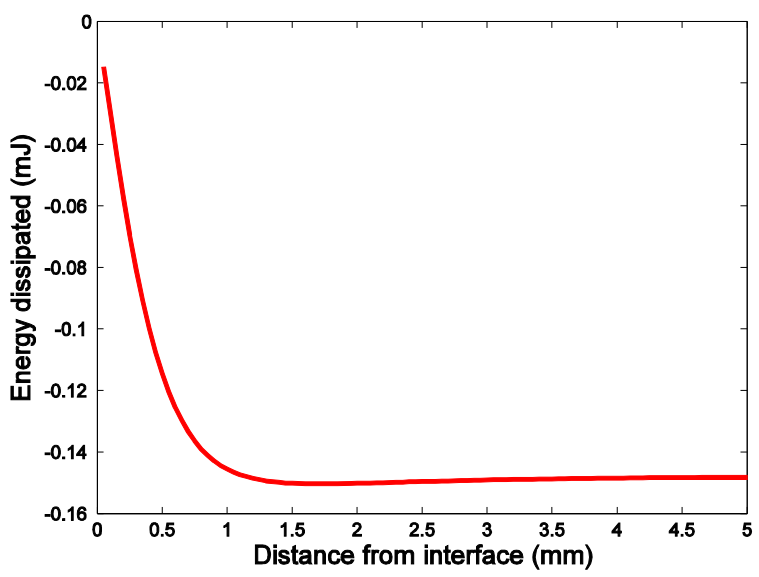

(b)

Figure 10: (a) Hysteresis curves for the points $y_{i}$ away from the contact centre; (b) Energy dissipated as a function of distance to the contact interface.

varying $Q / P$, unless the ratio $Q / P$ is raised close to the sliding limit $f$. For values of $Q / f P$ close to 1 , we expect that the coefficient obtained will not be very different to the near-sliding coefficient measured. For moderate and small value of $Q / f P$ the changes in the energy dissipated are very small, so that experimentally, this is unlikely to be a good method of determining the coefficient of friction with any great accuracy in this range.

\section{Discussion and conclusions}

Measurement of the coefficient of friction in partial slip contact is not an easy task. Some techniques for measuring the coefficient of friction have been suggested, but none has yet been very well established. The non-sliding "walking pad" test is conceptually very interesting. Experimentally, there are many challenges in this test. Particularly, the shear load transition from stationary to rigid body net displacement of the pad is difficult to determine precisely. However, the approach of using the rate of displacement and interpolating the experimental results using the 


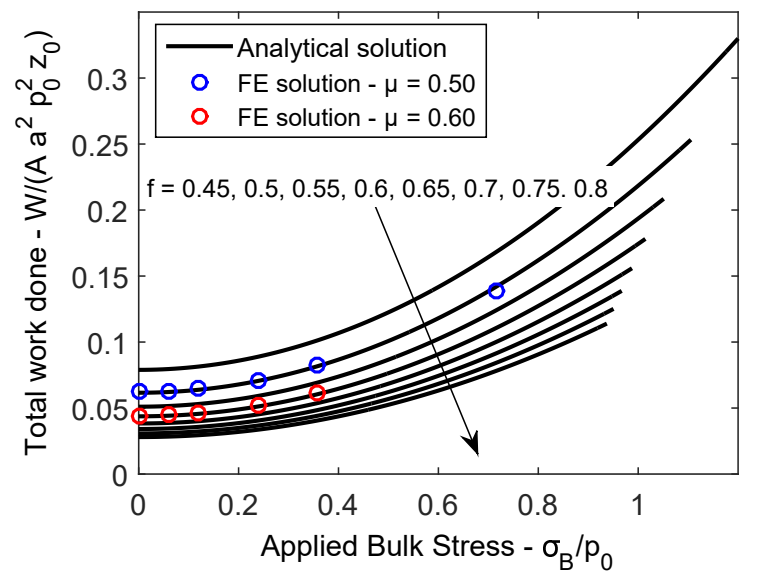

(a)

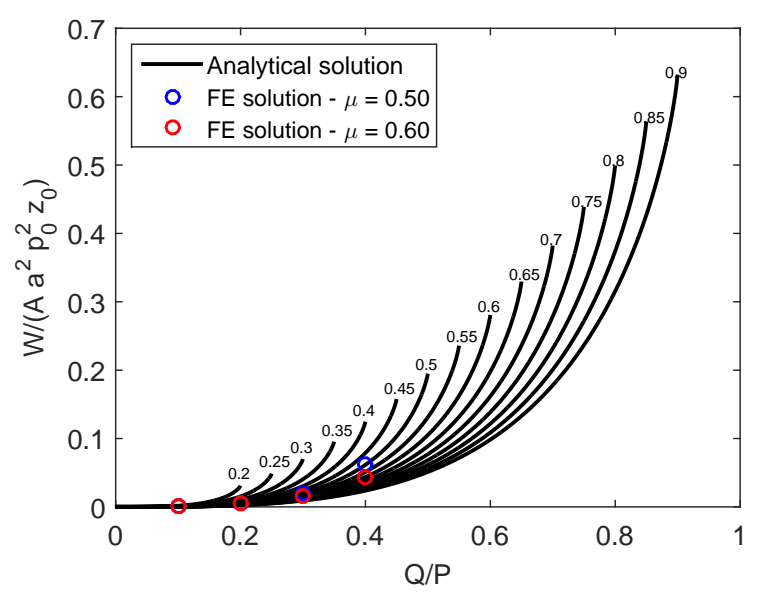

(b)

Figure 11: (a) Total work done as a function of the applied bulk stress $(Q / \mu P=0.6)$; (b) Energy Total work done as a function of the ratio $Q / P$.

semi-analytical solution is a good alternative. This test would also benefit greatly from a dedicated rig for measuring the coefficient of friction, with force and displacement sensors located closer to the contact.

The results for the coefficient of friction between Nickel superalloy single-crystal pads and polycrystalline specimens were higher than the coefficient of friction obtained with similar Nickel polycrystalline pads. This results were useful in the implementation of the "corrected" coefficient of friction for the correlation of near edge stresses with fretting fatigue life in [15]. However, due to the large scatter, particularly after each time the pad and specimen were reground, it is not possible to precisely estimate the coefficient of friction for this pair of material. It is also not apparent that there is a correlation between single-crystal orientation and friction at the interface, although the variation of the coefficient of friction with the crystal orientation in single-crystals have been reported previously [16]. Further analysis are required with a larger number of samples and controlled surface finishing to draw firm conclusions.

Energy based approaches for measuring the coefficient of friction has been used for the contact between spheres, and a study of an energy based approach for plane contacts was discussed here. Several factors may influence the energy dissipated in the contact, such as, the applied shear load and the bulk remote tension. It is possible to design an experimental procedure where one of these loads is varied and, by measuring the area of the curve for the remote displacement of a point and the applied tangential loads (i.e. the hysteresis loop of $Q-\delta$ ), the coefficient of friction may be calculated. Although the displacements in a 2D plane contacts do not converge to a finite value in the remote region, the total energy dissipated measured from the hysteresis loop of tangential force and remote displacements do converge. This is because the energy is dissipated only in the slip zones and, hence, provided that no other source of dissipation occur near the contact zone, the area inside the curve $Q-\delta$ must match the energy dissipated in the contact. However, this energy-based method is only valid if the other sources of energy dissipated in the contact are negligible [23, 24]. 
It is difficult to obtain an accurate measurement of the displacement near the contact zone and most rigs have a LVDT sensor very far from the contact. Note that, in an experimental rig, the further the measurement point moves away from the contact, the more likely it is that other sources of energy dissipation interfere with the measurements of the displacements. One solution for this problem could be using digital image correlation (DIC) to calibrate the LVDT sensors placed far from the contact. If a reliable measurement of the energy dissipation can be obtained, through the measurement of the area in the hysteresis loop, then the calculation of the coefficient of friction may be obtained from the total energy dissipated.

\section{Acknowledgements}

The authors would like to thank Rolls-Royce plc and EPSRC for the provision of the Dorothy Hodgkin Postgraduate Award and for the support throughout this work.

\section{References}

[1] O. Vingsbo, S. Söderberg, On fretting maps, Wear 126 (2) (1988) 131-147.

[2] D. A. Hills, D. Nowell, Mechanics of Fretting Fatigue, Kluwer Academic Publishers, United Kingdom, 1994.

[3] J. A. Araújo, D. Nowell, The effect of rapidly varying contact stress fields on fretting fatigue, Int. J. Fatigue 24 (7) (2002) 763-775.

[4] H. Murthy, G. Gao, T. N. Farris, Fretting fatigue of single crystal nickel at 600 c, Tribol. Int. 39 (10) (2006) 1227-1240.

[5] D. Dini, D. Nowell, Prediction of the slip zone friction coefficient in flat and rounded contact, Wear 254 (3) (2003) 364-369.

[6] K. Vadivuchezhian, S. Sundar, H. Murthy, Effect of variable friction coefficient on contact tractions, Tribol. Int. 44 (11) (2011) 1433-1442.

[7] C. Mary, S. Fouvry, J.-M. Martin, B. Bonnet, High temperature fretting wear of a ti alloy/cuniin contact, Surf. Coat. Technol. 203 (5) (2008) 691-698.

[8] D. Mulvihill, M. Kartal, A. Olver, D. Nowell, D. Hills, Investigation of non-coulomb friction behaviour in reciprocating sliding, Wear 271 (5) (2011) 802-816.

[9] S. Reina, R. J. H. Paynter, D. A. Hills, D. Dini, Determining the coefficient of friction between solids without sliding, Wear 269 (5) (2010) 339-343.

[10] S. Fouvry, P. Kapsa, L. Vincent, Analysis of sliding behaviour for fretting loadings: determination of transition criteria, Wear 185 (1) (1995) 35-46.

[11] R. D. Mindlin, H. Deresiewicz, Elastic spheres in contact under varying oblique forces, Trans. ASME Ser. E. J. Appl. Mech. 20 (1953) 372-344.

[12] S. Fouvry, P. Kapsa, H. Zahouani, L. Vincent, Wear analysis in fretting of hard coatings through a dissipated energy concept, Wear 203 (1997) 393-403.

[13] S. Fouvry, P. Kapsa, L. Vincent, Developments of fretting sliding criteria to quantify the local friction coefficient evolution under partial slip condition, Tribology Series 34 (1998) 161-172.

[14] A. Pasanen, A. Lehtovaara, R. Rabb, P. Riihimäki, Friction behavior of quenched and tempered steel in partial and gross slip conditions in fretting point contact, Wear 267 (12) (2009) 2200-2207.

[15] R. M. N. Fleury, R. J. H. Paynter, D. Nowell, The influence of contacting ni-based single-crystal superalloys on fretting fatigue of ni-based polycrystalline superalloys at high temperature, Tribol. Int. 76 (2014) 63-72.

[16] X. Huang, T. E. Gibson, M. Zhang, R. W. Neu, Fretting on the cubic face of a single-crystal ni-base superalloy at room temperature, Tribol. Int. 42 (6) (2009) 875-885.

[17] R. M. N. Fleury, Investigation of fretting fatigue in turbine fir tree blade to disc joints at high temperature, D.Phil. thesis, University of Oxford, Oxford, UK (2015). 
[18] C. A. Coulomb, Théorie des machines simples, Mémoires de Mthématique et de Ohysique de l'Académie Royale (1785) 161-342.

[19] C. Cattaneo, Sul contatto di due corpi elsatici: distribuzion local degli sfozi, Reconditi dell accademia nazionale dei lincei 27 (1938) 342-348,434-436,474-478.

[20] R. D. Mindlin, Compliance of elastic bodies in contact, J. Appl. Mech.-T ASME 16 (1949) 259-268.

[21] K. L. Johnson, Contact Mechanics, Cambridge University Press, 1985.

[22] D. Nowell, D. A. Hills, Mechanics of fretting fatigue tests, Int. J. Mech. Sci. 29 (5) (1987) 355-365.

[23] B. Berthel, A.-R. Moustafa, E. Charkaluk, S. Fouvry, Crack nucleation threshold under fretting loading by a thermal method, Tribology International 76 (2014) 35-44.

[24] A. R. Moustafa, Mésure thermographique des effets dissipatifs accompagnant le fretting: d'une construction rapide des cartes d'amorçage vers une meilleure comprehension des mécanismes d'endommagement, Ph.D. thesis, l'Ecole Centrale de Lyon, Écully, France (2016). 